\title{
Analysis of immunohistochemical expression of proinflammatory cytokines (IL-1 $\alpha$, IL-6, and TNF- $\alpha$ ) in gallbladder mucosa: comparative study in acute and chronic calculous cholecystitis
}

\author{
A. Kasprzak ${ }^{1}$, M. Szmyt ${ }^{1}$, W. Malkowski², W. Przybyszewska ${ }^{1}$, C. Helak-Łapaj ${ }^{3}$, \\ A. Seraszek-Jaros ${ }^{3}$, A. Surdacka ${ }^{4}$, A. Małkowska-Lanzafame ${ }^{4}$, E. Kaczmarek ${ }^{3}$ \\ ${ }^{1}$ Chair and Department of Histology and Embryology, Poznan University of Medical Sciences, Poznan, Poland \\ ${ }^{2}$ Chair of General Surgery T. Chałubinski Municipal Hospital in Ostrow Wielkopolski, Poland \\ ${ }^{3}$ Department of Bioinformatics and Computational Biology in the Chair of Clinical Pathomorphology, \\ Poznan University of Medical Sciences, Poznan, Poland \\ ${ }^{4}$ Department of Conservative Dentistry and Periodontology, Poznan University of Medical Sciences, Poznan, Poland
}

[Received 22 May 2014; Accepted 28 July 2014]

Background: Several studies have shown increased serum levels of proinflammatory cytokines (IL-1 $\alpha$, IL-6 and TNF- $\alpha$ ) in patients with cholelithiasis. The local expression of the proteins involved in pathogenesis of the disease is poorly recognised. Materials and methods: The authors examined immunohistochemically (IHC) the expression status of $I L-1 \alpha, I L-6$, and TNF- $\alpha$ in gallbladder mucosa of the patients with cholelithiasis as related to acute (ACC) and chronic (CCC) types of cholecystitis. Proinflammatory cytokines were quantitatively evaluated in gallbladder mucosa (epithelium and lamina propria) in ACC $(n=16)$ and CCC $(n=55)$ groups using modern spatial visualisation technique.

Results: Quantitative analysis of IHC signals showed no significant differences in IL-1 $\alpha$ and IL-6 immunoexpression in patients with ACC and CCC. A significantly greater IHC expression of TNF- $\alpha$ was detected in CCC as compared with ACC group. In either of the patient groups immunoexpression of IL-1 $\alpha$ and of TNF- $\alpha$ was significantly higher than that of IL-6. Immunoexpression of TNF- $\alpha$ was significantly higher than that of IL-1 $\alpha$ only in CCC group. A positive correlation was disclosed between IHC expression of IL-1 $\alpha$ and body mass index in CCC group. IHC expression of TNF- $\alpha$ correlated positively with expression of CD68 molecule (histiocytic marker), number of leukocytes in blood and higher grading of galIbladder wall in ACC group.

Conclusions: A more pronounced IHC expression of TNF- $\alpha$ and IL-1 $\alpha$ than IL-6 in both types of cholecystitis may suggest the role of these cytokines in pathogenesis of cholelithiasis. IHC expression of TNF- $\alpha$ shows better correlation with clinical/laboratory data in acute cholecystitis, and its quantitative prevalence over the remaining cytokines points to the role of the TNF- $\alpha$ in maintenance of inflammation in the course of cholelithiasis. (Folia Morphol 2015; 74, 1: 65-72)

Key words: cholelithiasis, cholecystitis, proinflammatory cytokines, immunohistochemistry, spatial visualisation method 


\section{INTRODUCTION}

The variables determining high risk of cholelithiasis $(\mathrm{CH})$ include female gender, obesity, insulin resistance, genetic conditioning and advanced age [20]. CH is most frequently accompanied by chronic cholecystitis (95\% cases), which leads to thickening of gallbladder wall with hypertrophy of muscularis externa and steatocholecystitis [13, 17]. Enhanced inflammation with an increased amount of fat in the gallbladder results in an abnormal wall structure and a decreased contractility [13]. It is worth stressing that $\mathrm{CH}$ is an important risk factor of galIbladder carcinoma [3, 22]. Several reports dealing with inflammation- and injury-associated cytokines in pathogenesis of $\mathrm{CH}$ are available in the literature [14, $15,21,24]$. The 3 cytokines: interleukin 1 (IL-1 $\alpha$ and IL-1 $\beta$ ), IL-6 and tumour necrosis factor-alpha (TNF- $\alpha$ ) are primarily secreted by monocytes and macrophages and represent potent multifunctional cytokines during immune response and inflammation, acting as proinflammatory proteins $[2,8,25]$. TNF- $\alpha$ is known to play an important role in initiation of inflammation by stimulating expression of other inflammatory mediators, including IL-6, what affects the absorption, secretion, and smooth muscle contractility of galIbladder [3, 4, 23]. IL-1 $\alpha$ is an intracellular cytokine with properties of both a cytokine and a transcription factor [2]. IL-6 trans-signalling is critically involved in the maintenance of the disease condition, by promoting transition from acute to chronic inflammation [18]. Lipopolysaccharide (LPS) stimulates release of IL-1, and IL-1 stimulates inflammation in guinea pig gallbladder in vivo [14]. Moreover, in an in vivo model IL-1 $\alpha$ and TNF- $\alpha$ were demonstrated to directly affect gallbladder epithelial cell absorptive and secreting function, which promoted development of gallstones [15]. In canine gallbladder application of a lithogenic diet was found to increase thickness of mucosa, myeloperoxidase activity, and IL-1 activity. Application of a bile obtained from dogs with pigment gallstones additionally decreased sodium absorption in normal pig gallbladder [16]. In the mouse model of $\mathrm{CH}$, obesity associated with intense consumption of purified carbohydrates was found to induce fat infiltration of gallbladder wall and to amplify local production of IL-1 $\alpha$ and TNF- $\alpha$. Obesity alone was noted to increase contents of free fatty acids and IL- 6 in gallbladder [12]. Serum of the patients with $\mathrm{CH}$ was found to manifest increased concentrations of IL-1, TNF- $\alpha$ and IL- 6 to the extent reflecting phase of inflammation
[24]. Gallbladder inflammation is an early feature of gallstone formation [15]. Moreover, inflammatory cytokines released in the gallbladder were found to initiate the induction of nitric oxide (NO) synthase, increased NO production, and inducing DNA damage in normal epithelial cell [10]. Application of a lithogenic diet and/or hunger in rodents increased turnover of mucosal cell and the desquamation of these cells into the lumen of the gallbladder [11]. The pathogenesis of $\mathrm{CH}$ and the relations of tissue expression of proinflammatory cytokines to calculous cholecystitis, steatocholecystitis and gallbladder carcinoma still remain to be precisely described $[3,22,23]$.

The authors of this study examined immunohistochemically (IHC) the expression status of IL- $1 \alpha, \mathrm{IL}-6$, and TNF- $\alpha$ in gallbladder mucosa of the patients with cholelithiasis as related to acute (ACC) and chronic (CCC) types of cholecystitis.

\section{MATERIALS AND METHODS}

\section{Patients}

All the patients were subjected to cholecystectomy in the T. Chałubiński Municipal Hospital in Ostrów Wielkopolski. The group with ACC $(n=16 ; 7$ women and 9 men; mean age $58 \pm 18$ years) and $\mathrm{CCC}(n=55$; 44 women and 11 men; mean age $32 \pm 14$ years) included patients who were diagnosed and subjected to surgery from 2003 to 2007. Exclusion criteria involved patients who had previously experienced other abdominal operations than cholecystectomy, patients with systemic diseases of connective tissue, patients with alcohol abuse, patients who chronically used anti-inflammatory drugs or other drugs which might modify the process of wound healing or inflammatory process itself. The available clinical data included age, gender and body mass index (BMI) and results of basic laboratory tests.

The number of gallstones was estimated using a semiquantitative scale: 1 (a single gallstone of any size), 2 ( 2 to 20 gallstones), 3 ( 21 to 100 gallstones), 4 (above 100 gallstones). Gallstones volume (in $\mathrm{cm}^{3}$ ) was estimated using the following formula: $V=1 / 6 \times$ $\times \pi \times A^{3}$; where $A$ is a diameter of gallstones.

In both groups of patients mostly mixed, cholesterol/ pigment/calcium gallstones were detected. However, their detailed chemical composition was not tested. Written informed consent was obtained from each patient before operation, and approval for the study was granted by the institution's Ethical Committee (No. 21/09). 


\section{Tissue material and microscopy image analysis}

The gallbladders were fixed in $10 \%$ buffered formalin and embedded in paraffin using the routine procedure. $\sim 5 \mu \mathrm{m}$ paraffin sections were placed on the SuperFrost/Plus microscope slides and stained with haematoxylin and eosin (HE). Histological alterations were examined independently by 2 histopathologists (AK, WB). Each tissue specimen was also evaluated using a simple numerical scoring system for the grade of lamina propria inflammation (G1) $(0-3)$ and the grade of muscularis externa/adventitia inflammation (G2) (0-3), in which 3 points denoted an intense and, most frequently, diffuse inflammatory infiltrate, 2 points referred to moderately intense but also diffuse inflammatory infiltrate, 1 point indicated individual, dispersed cells or focally arranged cells of inflammatory infiltrate, 0 points indicated tissue sections in which no cells of inflammatory infiltrate could be detected. The total grading in the entire wall of gallbladder $(G 1+G 2)$ was also evaluated. Thickness of the total wall of gallbladder was measured in $\mathrm{mm}$.

\section{Immunohistochemical studies}

To detect and to study the cellular localisation of IL- $1 \alpha$, IL- 6 and TNF- $\alpha$ IHC signal in gallbladder mucosa the classic $A B C$ (streptavidin-biotinylated peroxidase complex) IHC technique was used [5] with LSAB methodology (LSAB + System HRP, Dako, Glostrup, Denmark), described earlier. The individual stages of this technique were described in detail in our earlier reports [7]. Mouse anti-human monoclonal antibodies (MAbs) were employed, directed against IL-1 $\alpha$ (clone 4414), IL-6 (clone 6708.11) (both in dilution $1: 100$ ) (both from R\&D Systems Inc., UK), TNF- $\alpha$ (in dilution 1:50) (Abcam, Cambridge, UK), and against CD68 molecule in dilution 1:100 (clone PG-M1) (Dako) in each gallbladder tissue specimen. The sections were incubated with these primary MAbs at night at $4^{\circ} \mathrm{C}$, and afterwards were incubated with the LSAB + System HRP (Dako). The colour reaction was evoked with the HRP substrate, 0.05\% 3,3-diaminobenzidine tetrahydrochloride (DAB; Dako) in $0.05 \mathrm{M}$ Tris- $\mathrm{HCl}$ buffer, $\mathrm{pH} 7.6$, supplemented with $0.001 \% \mathrm{H}_{2} \mathrm{O}_{2}$. Following deparaffination and rehydration the preparations were additionally boiled in $10 \mathrm{mM}$ citrate buffer in a $700 \mathrm{~W}$ microwave oven for $18 \mathrm{~min}$ to increase antigen retrieval, washed in phosphate buffer saline and, then, subjected to the reaction according to the standard procedure. Every test was accompanied by a negative control in which specific antibodies were omitted and supplemented by a normal serum of a respective species in $0.05 \mathrm{M}$ Tris- $\mathrm{HCl}, \mathrm{pH} \sim 7.6$ supplemented with $0.1 \%$ bovine serum albumin and $15 \mathrm{mM}$ sodium azide.

For comparison of cytokine immunoexpression in gallbladder tissues, identical studies were performed in the archival tissue material from gingivae with chronic periodontitis $(\mathrm{n}=10)$ and reactive lymph nodes $(n=6)$ (positive control). Studies in the control group were not subjected to statistical analysis. Histological slides with the IHC expression of the cytokines were examined under the optical Olympus $\mathrm{BH}-2$ microscope coupled to a digital camera. Colour microscope images were recorded using $\times 40$ objective (at least 10 fields in every microscope slide with a positive IHC reaction), using LUCIA Image 5.0 computer software, documenting them in .jpg format on the computer hard disc.

\section{Microscopy image analysis}

Quantitative evaluation of the cytoplasmic IHC expression of the cytokines and CD68 was performed using image processing method based on spatial visualisation of markers in microscope images, elaborated and programmed in the A4D computer software $\mathrm{C}++$ language [6] and described in details in our previous paper [7]. Application of the technique of spatial visualisation to microscope images resulted in transformation of the flat image into a 3-dimensional image using its brightness as the third coordinate (the spatial coordinate). These so formed spatial images contain, apart from the examined objects, also other elements which had to be removed from the background level. For this purpose three filters were used: (1) filter of lucidity, (2) filter of colour, and (3) filter of saturation. The A4D programme allowed to calculate sequentially: (1) mean surface area of entire epithelium or entire mucosa (epithelium + lamina propria) in the recorded image (in pixels and $\mu \mathrm{m}^{2}$ ) for every patient and for the entire group of patients, (2) mean surface area of the IHC reaction (detecting contents of the cytokines) per the entire epithelial surface area or surface area of the entire mucosa (in pixels and $\mu \mathrm{m}^{2}$ ) for every patient and entire group of patients, (3) mean surface area of IHC reaction for a given cytokine per entire epithelial area or per surface area of entire mucosa in every patient and every group of patients, calculated and expressed also in per cents $(\%)[6,7]$. Results obtained using the A4D spatial visualisation software were compared with results of colour thresholding in HSI space obtained using Image J software (public domain, NHI, Bethesda, MD) and applied to the same images. For 
Table 1. Selected clinical data of patients with acute (ACC) and chronic calculous cholecystitis (CCC) (mean \pm standard deviation; median, minimum, maximum)

\begin{tabular}{lccc}
\hline Data & ACC $(\mathbf{n}=\mathbf{1 6})$ & CCC $(\mathbf{n}=\mathbf{5 5})$ & P \\
\hline Body mass index $\left[\mathrm{kg} / \mathrm{m}^{2}\right]$ & $27.96 \pm 3.28$ & $26.54 \pm 4.35$ & 0.289 \\
White blood cells $\left[\times 10^{9} / \mathrm{L}\right]$ & $13.84 \pm 4.48$ & $9.05 \pm 3.85$ & 0.001 \\
Width of gallbladder wall [mm] & $6.00 \pm 1.28$ & $5.59 \pm 2.03$ & 0.098 \\
G1* & Median: $2(\min 1, \max 3)$ & Median: $1(\min 0, \max 3)$ & 0.001 \\
G2* & Median: $3(\min 2, \max 3)$ & Median: $2(\min 1, \max 3)$ & $<0.001$ \\
G1+G2 & Median: $5(\min 3, \max 6)$ & Median: $3(\min 2, \max 6)$ & $<0.001$ \\
Number of gallstones ${ }^{*}$ & Median: $2(\min 1, \max 3)$ & Median: $2(\min 1, \max 4)$ & 0.046 \\
Volume of gallstones $\left[\mathrm{cm}^{3}\right]$ & $15.09 \pm 27.70$ & $16.48 \pm 23.91$ & 0.207 \\
\end{tabular}

$\mathrm{G} 1$ - the grade of lamina propria inflammation; $\mathrm{G} 2$ - the grade of muscularis externa/adventitia inflammation; $\mathrm{G} 1+\mathrm{G} 2$ - total grading in the entire wall of gallbladder; ${ }^{*}$ Score see Materials and Methods

this purpose, the Color Thresholder plug, available at http://rsb.info.nih.gov/ij web page, was installed in the basic version of the software. The error of measuring expression of IHC reaction was appraised by evaluation of the standard error of differences between measurements conducted on the same 250 microscope images but obtained using two distinct techniques (A4D and Image J). The standard error of mean measurement value amounted to 97 pixels or $0.97 \%$ of the mean area of reaction established by the spatial visualisation technique. Thus, it might be accepted that error of the method did not exceed $1 \%$.

Results obtained in the two softwares for image analysis (LUCIA Image 5.0 and A4D) were exported to the format of Microsoft Excel programme which is compatible with the Statistica PL v. 8 programme (Statsoft, Inc., Tulsa, OK, USA), with the aim to conduct statistical analysis. The results related to expression of cytokines and CD68 were presented as area fraction (percentage) of the IHC reactions manifested by gallbladder mucosa (epithelium + lamina propria).

\section{Statistical analysis}

The Shapiro-Wilk normality test was performed to determine whether the obtained results were normally distributed. A significance of differences between ACC and CCC groups was evaluated using the $t$ test. Subsequently, parameters of a descriptive statistics were calculated (arithmetical mean, standard deviation, median value, minimum and maximum values). Results of microscopic image analysis were compared between the ACC and CCC independent group of the patients using the Mann-Whitney $U$ test. The Wilcoxon test was used for non-parametric dependent data. Correlations between data rows were determined employing Spearman's rank correlation index. Differences and relationships were accepted to be statistically significant at the level of $p<0.05$.

\section{RESULTS}

As expected, a significantly more intense inflammation (grading) (G1, G2 and G1 + G2) was observed in gallbladders of patients with ACC than in those with CCC. Moreover, the number of leukocytes (white blood cells [WBC]) in the blood was significantly higher in ACC group. No difference between groups was disclosed in mean thickness of gallbladder wall. Patients with CCC had higher number of gallstones, but the mean volumes of the gallstones were similar in ACC and CCC group (Table 1).

\section{IL-1 $\alpha$, IL-6 and TNF- $\alpha$ immunoexpression in gallbladder mucosa: cellular localisation}

Immunohistochemical expression of IL- $1 \alpha$ and TNF- $\alpha$ was detected in all patients (100\%) of both groups. IL-6 expression was demonstrated in $62 \%$ cases of ACC and $63 \%$ cases of CCC.

Irrespectively of the patient group (ACC, CCC), IHC expression of all proinflammatory cytokines pertained different types of cells in gallbladder mucosa (epithelial cells, subepithelial cells in lamina propria). The IHC positive reaction was observed mostly in cell cytoplasm (Fig. 1A-F), but immunopositive cell membranes were also evident, especially in epithelial cells filled with vacuoles of mucins (Fig. 1A, B).

Interestingly, immunoexpression of all 3 cytokines was demonstrated in columnar cells of gallbladder 


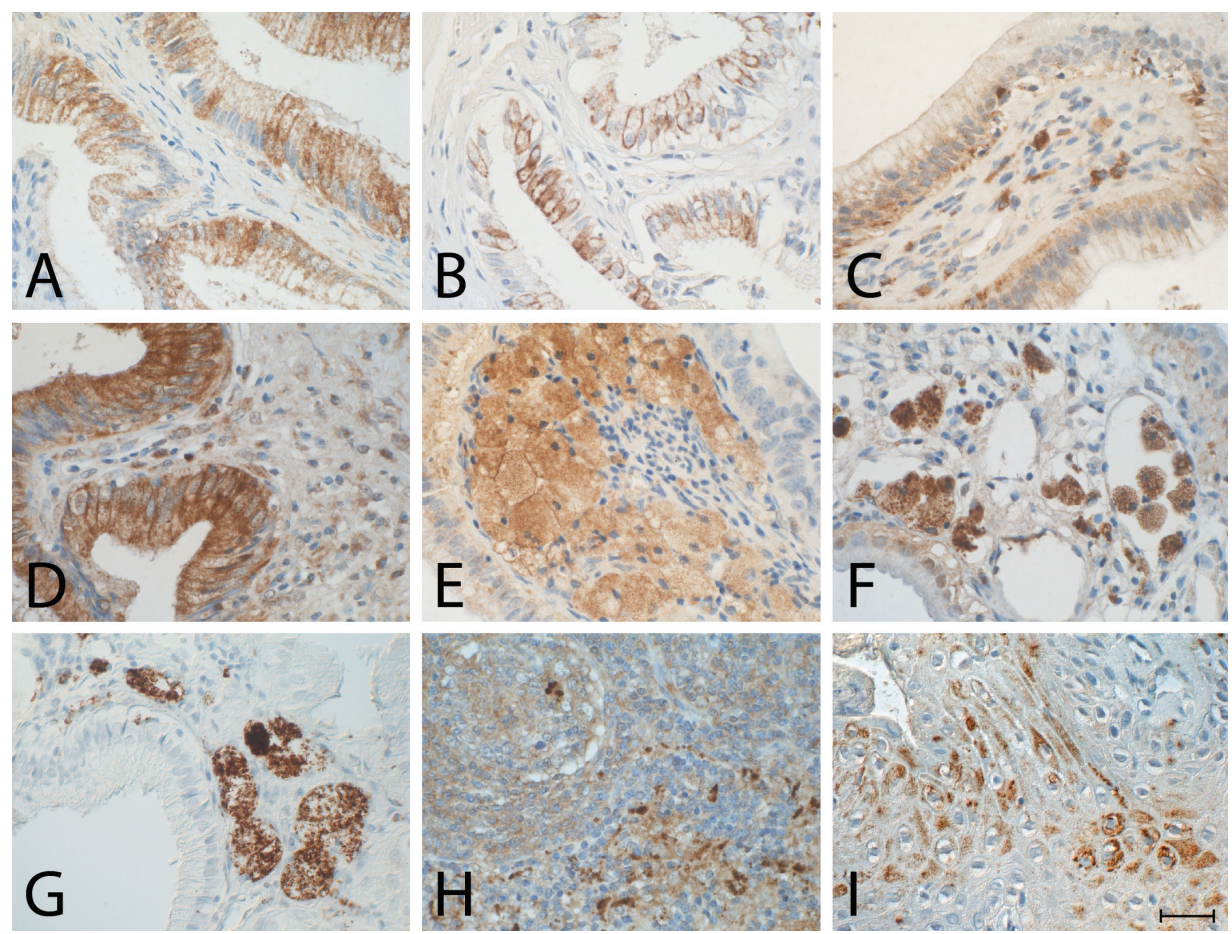

Figure 1. Immunohistochemical expression of proinflammatory cytokines in gallbladder mucosa of the patients with cholelithiasis. A, C. Interleukin (IL)-1 $\alpha$; B. IL-6; D. Tumour necrosis factor-alpha (TNF- $\alpha$ ) expression in columnar epithelial cells in gallbladder mucosa of the patients with chronic calculous cholecystitis; IL-1 $\alpha$ (C) and TNF- $\alpha$ (D) expressions present also in inflammatory cells in lamina propria (mostly macrophages); IL-1 $\alpha(\mathbf{E})$ and TNF- $\alpha(\mathbf{F})$ expressions in foamy cells (macrophages) under epithelium of gallbladder; CD68 (G) expression in histiocytic macrophages in the same patient as in Figure 1F; H. TNF- $\alpha$ expression in lymphoid cells of reactive lymph node (positive control); I. IL-6 localisation in keratinocytes of gingivae in chronic periodontitis (positive control); ABC technique; haematoxylin counterstained; bar $=50 \mu \mathrm{m}$ in all cases.

Table 2. Comparative analysis of immunocytochemical tissue expression (\% of IHC reaction) of the proinflammatory cytokines in gallbladder mucosa of the patients with acute (ACC) and chronic (CCC) type of calculous cholecystitis (mean \pm standard deviation)

\begin{tabular}{lccc}
\hline Cytokine & ACC & CCC & P \\
\hline Interleukin- $1 \alpha$ (IL-1 $\alpha)$ & $5.26 \pm 4.32^{*}$ & $5.07 \pm 6.22^{* * *}$ & 0.349 \\
Interleukin-6 (IL-6) & $1.02 \pm 1.76$ & $1.85 \pm 2.58$ & 0.504 \\
Tumour necrosis factor $\alpha($ TNF- $\alpha)$ & $6.89 \pm 8.65^{*}$ & $13.13 \pm 8.94^{* *}$ & 0.006 \\
\hline
\end{tabular}

$\mathrm{p}$ - level of significance; ACC group: * $\mathrm{p}<0.01$ between IL-1 $\alpha$ and IL- 6 and TNF- $\alpha$ and IL- 6 expression; CCC group: ${ }^{* *} \mathrm{p}<0.001$ between TNF- $\alpha$ and both IL- $1 \alpha$ and IL- 6 expression; ${ }_{* * *} p<0.01$ between IL- $1 \alpha$ and IL-6 expression

epithelium in both groups of patients (Fig. 1A-D) and, moreover, for IL- $1 \alpha$ and IL-6 this localisation prevailed. An intense positive IHC signal for IL- $1 \alpha$ and TNF- $\alpha$ was noted also in CD68-positive macrophages, scattered in gallbladder lamina propria (Fig. 1C, D) or gathered in the form of foamy cells (Fig. 1E, F). The exemplary $\mathrm{IHC}$ expression of the macrophage histiocytic marker is illustrated in Figure 1G. Occasionally, the IHC positive reaction on IL- $1 \alpha$ and TNF- $\alpha$ was observed in endothelial cells and tubuloacinar glands (data not shown).

In the positive control, IL- $1 \alpha$, IL- 6 and TNF- $\alpha$ immunoexpression involved both lymphoid cells in reactive lymph node (Fig. 1H), and keratinocytes of the gingival stratified squamous epithelium (Fig. 11).

\section{Quantitative analysis of IL- $1 \alpha$, IL- 6 and TNF- $\alpha$ immunoexpression in gallbladder mucosa}

Mean value of IL- $1 \alpha$ immunoexpression in galIbladder mucosa of the patients with ACC and CCC was similar $(p>0.05)$. Mean IHC expression of IL-6 was low (less than $2 \%$ of reaction) and also showed no significant intergroup difference $(p>0.05)$. Mean IHC expression of TNF- $\alpha$ in ACC was significantly lower than that in CCC group $(p<0.01)$ (Table 2, Fig. 2). 


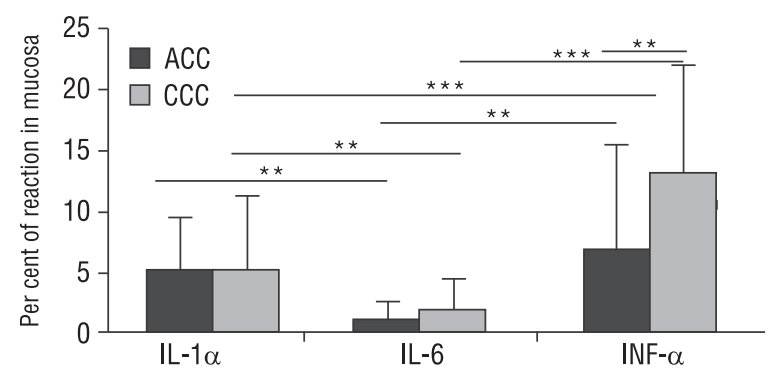

Figure 2. Comparative immunohistochemical expressions of interleukin (IL)- $1 \alpha$, IL-6 and tumour necrosis factor-alpha (TNF- $\alpha$ ) in acute (ACC, $n=16$ ) and chronic type of calculous cholecystitis (CCC, $\mathrm{n}=55$ ); ${ }^{* * *} \mathrm{p}$ (level of significance) value $<0.001 ;{ }^{* *} \mathrm{p}<0.01$.

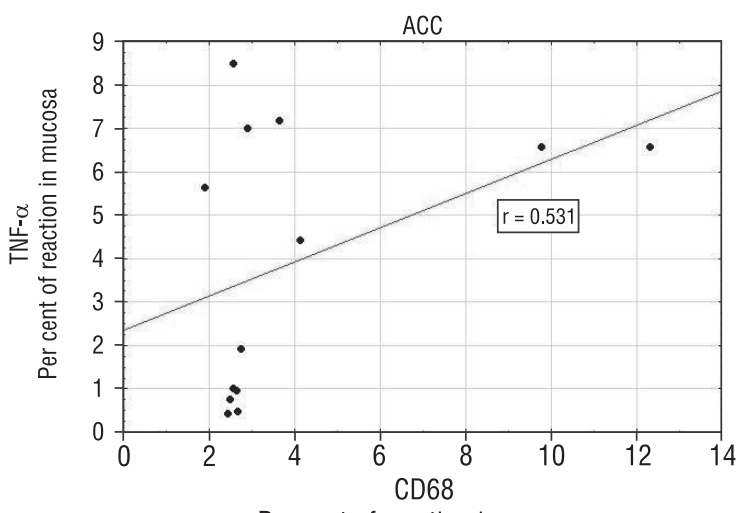

Per cent of reaction in mucosa

Figure 3. A positive correlation between immunohistochemical expressions of tumour necrosis factor-alpha (TNF- $\alpha$ ) and the marker of histiocytic macrophages (CD68 molecule) in acute calculous cholecystitis (ACC) group $(\mathrm{p}<0.05)$.

In either of the patient groups IHC expression of IL- $1 \alpha$ and of TNF- $\alpha$ was significantly higher than that of IL-6. ACC group showed a similar IHC expression of IL- $1 \alpha$ and TNF- $\alpha$ and a very poor immunoexpression of IL-6. In CCC group IHC expression of TNF- $\alpha$ expression quantitatively prevailed and it was higher than immunoexpression of either IL-1 $\alpha$. The least pronounced IHC expression in this group of patients involved IL- 6 (Table 2, Fig. 2).

Relationship between IL- $1 \alpha$, IL- 6 and TNF- $\alpha$ reciprocal immunoexpression

Positive correlation was documented between immunoexpressions of TNF- $\alpha$ and the CD68 molecule in ACC group ( $r=0.531$ ) (Fig. 3). In CCC group correlation between immunoexpressions of TNF- $\alpha$ and that of CD68 molecule was not statistically significant $(r=0.197 ; p>0.05)$ (data not shown).
There were no correlations between reciprocal immunoexpression of the IL- $1 \alpha$ and IL- $6(r=0.211$ in ACC; $r=-0.069$ in CCC), IL- $1 \alpha$ and TNF- $\alpha(r=0.445$ in ACC; $r=0.050$ in CCC), and IL- 6 and TNF- $\alpha(r=0.220$ in ACC; $r=0.337$ in CCC) ( $p>0.05$ for all cases) (data not shown).

Tissue immunoexpression of IL-1 $\alpha$, IL- 6 and TNF- $\alpha$ and selected clinical/laboratory data

In ACC patients no significant correlation could be detected between immunoexpression of IL-1 $\alpha$ and IL-6 on one hand and clinical data or intensity of inflammatory activity (grading) in gallbladder on the other. Moreover, in this group immunoexpression of TNF $\alpha$ was found to have direct relationship with WBC number and it showed a highly positive correlation with the total grading in gallbladder $(\mathrm{G} 1+\mathrm{G} 2)$.

In patients with CCC a weak positive correlation was observed between IL-1 $\alpha$ immunoexpression and patients' BMI. The intensity of IL-6 immunoexpression showed also very weak negative correlations with grading in mucosa (G1) and with total inflammatory activity in gallbladder $(\mathrm{G} 1+\mathrm{G} 2)$. Also a weak negative correlation was detected between IL- 6 immunoexpression and WBC number in this group of patients. Surprisingly, in this group of patients the immunoexpression of TNF- $\alpha$ demonstrated a negative correlation with grading (G2, G1+G2) (Table 3).

\section{DISCUSSION}

The inflammatory response in $\mathrm{CH}$ is associated with increases in myeloperoxidase and IL-1-like activities in the gallbladder wall [15]. In inflammatory lesions of gallbladder mucosa induced with a lithogenic bile a decreased sodium absorption by epithelium was noted [16]. This study have documented mainly epithelial localisation of IL- $1 \alpha$ and IL- 6 immunoexpression. Expression of IL-1 $\alpha$, more frequently than expression of IL-6, was detected also in mononuclear cells of inflammatory infiltrates, mainly in macrophages. Surprisingly, no differences in intensity of immunoexpression (as area fraction of the IHC reaction in gallbladder mucosa) of both interleukins have been disclosed between types of cholecystitis (acute vs. chronic). The cytoplasmic IHC expression of TNF- $\alpha$ was detected also in epithelial cells of gallbladder, but the most numerous cell population with IHC expression of the cytokine involved CD68-positive tissue macrophages. The positive correlation between immunoexpressions of TNF- $\alpha$ and CD68 molecule 
Table 3. Spearman's rank correlation coefficients ( $r$ ) between immunocytochemical expression of cytokines in gallbladder mucosa and selected clinical and laboratory data in patients with acute (ACC) and chronic (CCC) type of calculous cholecystitis

\begin{tabular}{|c|c|c|c|c|c|c|}
\hline \multirow[t]{2}{*}{ Clinical data } & \multicolumn{3}{|c|}{ ACC } & \multicolumn{3}{|c|}{ CCC } \\
\hline & IL-1 $\alpha$ & IL-6 & TNF- $\alpha$ & IL-1 $\alpha$ & IL-6 & TNF- $\alpha$ \\
\hline Age [years] & 0.062 & 0.348 & -0.007 & 0.071 & 0.106 & 0.209 \\
\hline Body mass index $\left[\mathrm{kg} / \mathrm{m}^{2}\right]$ & 0.265 & 0.053 & 0.270 & 0.368 & -0.066 & -0.020 \\
\hline White blood cells $\left[\times 10^{9} / \mathrm{L}\right]$ & 0.381 & 0.390 & 0.591 & 0.175 & -0.382 & -0.109 \\
\hline Number of gallstones* & -0.223 & -0.305 & 0.096 & -0.169 & -0.142 & -0.125 \\
\hline Volume of gallstones $\left[\mathrm{cm}^{3}\right]$ & -0.319 & -0.390 & -0.318 & -0.190 & -0.210 & -0.179 \\
\hline Width of gallbladder wall [mm] & 0.184 & 0.328 & 0.086 & 0.079 & 0.025 & -0.042 \\
\hline $\mathrm{G} 1^{*}$ & 0.090 & 0.319 & 0.425 & 0.219 & -0.342 & -0.241 \\
\hline $\mathrm{G} 2^{*}$ & 0.424 & 0.390 & 0.459 & -0.024 & -0.172 & -0.414 \\
\hline $\mathrm{G} 1+\mathrm{G} 2^{*}$ & 0.290 & 0.435 & 0.535 & 0.135 & -0.321 & -0.414 \\
\hline
\end{tabular}

IL — interleukin; TNF- $\alpha$ - tumour necrosis factor-alpha; G1 — the grade of lamina propria inflammation; $\mathrm{G} 2$ — the grade of muscularis externa/adventitia inflammation; $\mathrm{G} 1+\mathrm{G} 2$ — total grading in entire gallbladder wall; * Score see Materials and Methods; bold numbers indicate significantly different values $(p<0.05)$

(a marker of histiocytic macrophages) was noted only in ACC group. A higher IHC expression of TNF- $\alpha$ was detected in the patients with chronic as compared to those with acute cholecystitis, but only in acute cholecystitis positive correlations were seen between TNF- $\alpha$ immunoexpression on one hand and leukocytes number and total grading in gallbladder wall on the other. This may point to proinflammatory role of TNF- $\alpha$ and to activation of the lymphokine production (mainly in macrophages) in the acute phase of inflammation. The results of this study have been consistent with those reports which documented localisation of IL-1 producers: cellular sources of IL-1 involved mainly monocytes and macrophages, and out of other cell types epithelial cells and keratinocytes were mentioned [2]. The production of IL- 6 and TNF- $\alpha$ was demonstrated also in intrahepatic biliary epithelial cells [25]. The most potent IL-1 synthesis-inducing factors include LPS of Gram-negative bacteria [1]. Also in the patients with $\mathrm{CH}$ included in this study, influence of a bacterial factor on induction of the cytokine production cannot be excluded. Molecular analyses described 2 IL-1 molecules of a different molecular weight, depending on location of the cytokine. In our gallbladder tissue sections, the cytoplasmic location of the cytokine has been documented, which has indicated the presence of the $31 \mathrm{kDa}$ precursor form (prolL-1 $\alpha$ ) [2]. In cases of a more pronounced production of the IL- $1 \alpha$ form in gallbladder epithelial cells as compared with the production of IL-6 in our patients, a double role of the cytokine may be suggested. It may be responsible for a disturbed absorptive function of the cells and may promote lithogenesis, as described in the animal model [16]. Moreover, the production of the cytokine may reflect terminal differentiation of gallbladder epithelium. The increased local expression, through an autocrine and/or paracrine action may play a protective function in cholecystitis.

IL-6 is a proinflammatory cytokine (produced by various cell types) with pleiotropic biological functions, including induction of the hepatic acute phase response and differentiation of activated B cells into lg-secreting plasma cells [8]. Overexpression of IL-6 itself was described in a number of solid tumours and in the hyperproliferative lesions of psoriasis [9]. The role played by IL- 6 in damaging intercellular junctions, changes in epithelial cell phenotype, in disturbing absorptive and secretory properties of gallbladder epithelial cells in $\mathrm{CH}$ requires further studies, encompassing also other components of the IL-6/IL-6R system.

In CCC group this study confirmed a significant (even if rather weak) direct relationship between intensity of IL- $1 \alpha$ immunoexpression and BMI. Other investigators described that both obesity and dietary carbohydrates increase gallbladder total fat, triglycerides, cholesterol, TNF- $\alpha$, and IL- $1 \alpha$. The data suggest that obesity also increases gallbladder free fatty acids and IL-6 in gallbladder [12].

The role of TNF- $\alpha$ in pathogenesis of $\mathrm{CH}$ seems to be very important. The production of mRNA (but not protein) for TNF- $\alpha$ was demonstrated in untreated mouse gallbladder epithelial cells [19]. Upon treatment with LPS, these cells synthesised and secreted TNF- $\alpha$ (mRNA and protein), and produced also mRNA for IL-1 $\alpha$ and IL-6 [19]. In this study, a significantly higher immunoexpression of TNF- $\alpha$ was demonstra- 
ted in gallbladders of patients with CCC as compared to cases with acute cholecystitis. Positive correlation was documented between immunoexpressions of TNF- $\alpha$ and the CD68 molecule in ACC group. This observation suggests that histiocytic macrophages are the main source of TNF- $\alpha$, particularly in the acute type of calculous cholecystitis.

In chronic type of cholecystitis, immunoexpression of TNF- $\alpha$ was also higher as far as the 3 proinflammatory cytokines are concerned. In this type of cholecystitis, cellular production of TNF- $\alpha$ was observed in a broader phenotypic panel of cells.

\section{CONCLUSIONS}

The significantly higher IHC expression of TNF- $\alpha$ and IL- $1 \alpha$ as compared with that of IL- 6 in both types of cholecystitis (ACC and CCC) may suggest a more pronounced role of local IHC expression of TNF- $\alpha$ and $\mathrm{IL}-1 \alpha$ in pathogenesis of $\mathrm{CH}$. Immunoexpression of studied cytokines in epithelial gallbladder cells may confirm their role in pathogenesis of $\mathrm{CH}$ also in in vivo conditions (lost function of epithelial cells and/ /or stimulation of mucin production). Localisation of TNF- $\alpha$ in CD68-positive tissue macrophages confirms the mainly proinflammatory function of the cytokine, particularly in the acute type of calculous cholecystitis. IHC expression of TNF- $\alpha$ shows better correlation with clinical and laboratory data in acute type of cholecystitis, and its quantitative prevalence over the remaining cytokines points to the role of the cytokine in maintenance of inflammation in the course of $\mathrm{CH}$.

\section{ACKNOWLEDGEMENTS}

The second author (MS) received grant within Subaction 8.2.2. "Regional Strategies of Innovation", Action 8.2 "Transfer of knowledge", Priority VIII "Regional Staff of Management", Operative Programme of Human Capital, co-financed by the European Social Fund of the European Union and by the state budget.

\section{REFERENCES}

1. Arend WP, Massoni RJ (1986) Characteristics of bacterial lipopolysaccharide induction of interleukin 1 synthesis and secretion by human monocytes. Clin Exp Immunol, 64: 656-664.

2. Dinarello CA (2006) Interleukin 1 and interleukin 18 as mediators of infammation and the aging process. Am J Clin Nutr, 83: 447S-455S.

3. Gilloteaux J, Tomasello LM, Elgison DA (2003) Lipid deposits and lipo-mucosomes in human cholecystitis and epithelial metaplasia in chronic cholecystitis. Ultrastruct Pathol, 27: 313-321.
4. Hotamisligil GS, Shargill NS, Speigelman BM (1993) Adipose expression of tumor necrosis factor-alpha: direct role in obesity-linked insulin resistance. Science, 259: 87-91.

5. Hsu SM, Raine L, Fanger H (1981) Use of avidin-biotin-peroxidase complex $(A B C)$ in immunoperoxidase techniques: a comparison between $A B C$ and unlabeled antibody (PAP) procedures. J Histochem Cytochem, 29: 577-580.

6. Kaczmarek E, Strzelczyk R (2005) From two to three-dimensional visualisation of structures in light and confocal microscopy applications for biomedical studies. In: Mendez-Vilas A, Labajos-Broncano L eds. Current issues on multidisciplinary microscopy research and education. FORMATEX microscopy book series no. II Formatex Research Centre, Badajoz, pp. 289-295.

7. Kasprzak A, Malkowski W, Helak-Łapaj C, Seraszek A, Kaczmarek E, Adamek A, Zabel M (2010) Polysaccharides and mucin 5AC (MUC5AC) expression in gallbladder mucosa of young patients with gallstones as evaluated by spatial visualization and quantification. Folia Histochem Cytobiol, 48: 646-657.

8. Kishimoto T (1989) The biology of interleukin-6. Blood, 74: 1-10.

9. Kishimoto T (2010) IL-6: from its discovery to clinical applications. Int Immunol, 22: 347-352.

10. Kitasato A, Tajima Y, Kuroki T, Tsutsumi R, Adachi T, Mishima T, Kanematsu $T$ (2007) Inflammatory cytokines promote inducible nitric oxide synthase-mediated DNA damage in hamster gallbladder epithelial cells. World J Gastroenterol, 13: 6379-6384.

11. Marsch-Ziegler U, Palme G (1982) Effect of a lithogenic diet and starvation on the regeneration of gallbladder epithelium. Leber Magen Darm, 12: 157-161.

12. Mathur A, Al-Azzawi HH, Lu D, Yancey KW, Swartz-Basile DA, Nakeeb A, Pitt HA (2008) Steatocholecystitis: the influence of obesity and dietary carbohydrates. J Surg Res, 147: 290-297.

13. Pitt HA (2007) Hepato-pancreato-biliary fat: the good, the bad and the ugly. HPB (Oxford), 9: 92-97.

14. Prystowsky JB, Rege RV (1997) Interleukin-1 mediates guinea pig gallbladder inflammation in vivo. J Surg Res, 71: 123-126.

15. Rege RV (2000) Inflammatory cytokines alter human gallbladder epithelial cell absorption/secretion. J Gastrointest Surg , 4: 185-192.

16. Rege RV, Prystowsky JB (1996) Inflammatory properties of bile from dogs with pigment gallstones. Am J Surg, 171: 197-201.

17. Rosai J (2004) Gallbladder and extrahepatic bile ducts. In: Rosai J ed. Rosai and Ackerman's Surgical Pathology, Mosby, Edinburgh, London, New York, Oxford, Philadelphia, St. Louis, Sydney, Toronto, pp. 1035-1055.

18. Rose-John S, Scheller J, Elson G, Jones SA (2006) Interleukin-6 biology is coordinated by membrane-bound and soluble receptors: role in inflammation and cancer. J Leukoc Biol, 80: 227-236.

19. Savard CE, Blinman TA, Choi HS, Lee SK, Pandol SJ, Lee SP (2002) Expression of cytokine and chemokine mRNA and secretion of tumor necrosis factor-alpha by gallbladder epithelial cells: response to bacterial lipopolysaccharides. BMC Gastroenterol 2: 23 (doi: 10.1186/1471-230X-2-23)

20. Schirmer BD, Winters KL, Edlich RF (2005) Cholelithiasis and cholecystitis. J Long Term Eff Med Implants, 15: 329-338.

21. Sehgal PB (1996) Interleukin-6-type cytokines in vivo: regulated bioavailability. Proc Soc Exp Biol Med, 213: 238-247.

22. Tazuma S, Kajiyama G (2001) Carcinogenesis of malignant lesions of the gallbladder. The impact of chronic inflammation and gallstones. Langenbecks Arch Surg, 386: 224-229.

23. Tsai CJ (2009) Steatocholecystitis and fatty gallbladder disease. Dig Dis Sci, 54: 1857-1863.

24. Tsaregorodtseva TM, Zotina MM, Serova TI, Sokolova GN, Iakimchuk GN (2003) Interleukins in chronic diseases of digestive organs. Ter Arkh, 75: 7-9.

25. Yasoshima M, Kono N, Sugawara H, Katayanagi K, Harada K Nakanuma $Y$ (1998) Increased expression of interleukin-6 and tumor necrosis factor-alpha in pathologic biliary epithelial cells. Lab Invest, 78: 89-100. 